
Sy
Volume and Issues Obtainable at Center for Sustainability Research and Consultancy
Journal of Accounting and Finance in Emerging Economies
ISSN: 2518-0318 ISSN (E) 2518-8488
Volume 6: Issue 2 June 2020
Journal homepage: www.publishing.globalcsrc.org/jafee

\title{
Prevalence of Poor Sleep and Relationship of Physical Exercise with Sleep among Male Adult Exercisers
}

\author{
${ }^{1}$ Asif Ali, ${ }^{2}$ Muhammad Azam, ${ }^{3}$ Faisal Mehmood
}

${ }^{1}$ Associate Professor, Department of Physical Education and Sports Sciences, Government College University, Lahore Pakistan, goraya128@ hotmail.com

${ }^{2}$ Assistant Professor, Department of Physical Education and Sports Sciences, Government College University, Lahore Pakistan, goraya78@gmail.com

${ }^{3} \mathrm{PhD}$ Scholar, Lahore University, Lahore Pakistan, ravian.gold@gmail.com

\section{ARTICLE DETAILS \\ History \\ Revised format: May 2020 \\ Available Online: June 2020 \\ Keywords \\ Exercise, sleep, health, sports, participation}

\section{JEL Classification}

IO, II

\begin{abstract}
Physical exercise has been suggested to show beneficial effects for various diseases and also for sleep. This study aims to assess the prevalence of sleep problems, and the relationship of physical exercise with sleep health among adult male exercisers. A total of 199 randomly selected athletes (males) took part in this study. All participants responded to a questionnaire consisted of items related to demographic information that also included sports participation level and playing experience. Self-reported sleep quality and physical exercise were using PQSI and three items concerning exercise frequency, intensity, and duration. The analysis revealed following main findings: 1) a significant negative correlation between physical exercise and sleep medication, 2) a significantly negative relationship between sports/ exercise participation level and sleep disturbance, 3) a 3.7 average global score of PSQI in the total sample, 4) Exercise frequency was negatively correlated with daytime dysfunction whereas exercise intensity was negatively correlated with sleep disturbance and sleep efficiency but positively correlated with daytime dysfunction, 5) and prevalence of poor sleep in $22 \%$ participants. There is some possibility to suggest that exercise may improve sleep health among adult male exercisers. The increased exercise frequency has a more positive impact to improve sleep but the relationship between exercise intensity and sleep remains inconclusive. The existence of sleep problems in the athletic population essentially requires further attention for reducing the prevalence of poor sleep using other approaches along with continuous exercise for good sleep.
\end{abstract}

\section{OPEN ACCESS}

(C) 2020 The authors, under a Creative Commons Attribution-

NonCommercial 4.0

Corresponding author's email address: goraya78@gmail.com

Recommended citation: Ali, A., Azam, M. \& Mehmood, F. (2020). Prevalence of Poor Sleep and Relationship of Physical Exercise with Sleep among Male Adult Exercisers. Journal of Accounting and Finance in Emerging Economies, 6(2), 539-545 


\section{Introduction and Literature Review}

Physical exercise has been extensively suggested as an aiding mean to enhance health and well-being in the various populations such as children, adolescents, adults, old aged persons and in the wide range populations with various physical diseases including cardiovascular diseases, obesity, diabetes, cancer (Anderson \& Durstine, 2019), and psychiatric conditions including depression, self-harm, stress, suicidality, and substance abuse (Zschucke, Gaudlitz, \& Ströhle, 2013). Increased level of physical exercise recommended to boost affective states, reduce pain, prevent obesity, and to improve sleep quality in persons exhibiting conditions of insomnia (Mork et al., 2013).

An adequate level of sleep also has been considered essential for optimal physical and cognitive functioning. Evidence suggests that inadequate and abnormal sleep patterns are considered among the major factors contributing to many health problems such as diabetes, obesity, and depression, and hence believed as a serious health concern (Bhaskar, Hemavathy, \& Prasad, 2016; Singareddy et al., 2012). Even some reports showed decreased rates of mortality as well as morbidity in individuals with increased sleep quality. Prevalence of sleep-related disorders has increased remarkably during recent years across the globe and varies depending upon geographical region, age, gender, physical, and psychiatric conditions, environment and socioeconomic status of the populations (Cao et al., 2017). Approximately $30 \%$ of general population reported experiencing some type of sleep problems, and even some studies reported a higher level of prevalence (Bhaskar et al., 2016). In particular, the rate of disturbed sleep is higher in young adults, females, and patients with psychiatric and medical conditions (Cao et al., 2017). Keeping in view the prevalence and negative consequences of poor sleep, interventions that help to prevent and treat sleep problems have become the topic of priority for clinicians and professionals in the public health domain.

Physical exercise considered as non-invasive and complementary medicine to treat many diseases and also to improve sleep in adults. Physical exercise refers to the planned and structured physical activities performed in view of a certain duration, frequency, and intensity (Caspersen, Powell, \& Christenson, 1985). Moderate intensity exercise results in raising the breathing more than normal whereas vigorous exercises lead to fast breathing during which speaking is difficult. Previous research showed beneficial effects of physical exercise for sleep comparing exercisers and nonexercisers. The focus of this study is to investigate how exercise affects sleep in regular exercisers. Another purpose was to determine the prevalence of sleep problems in adult exercisers.

\section{Methods}

\subsection{Participants}

A total of 223 adults with ages ranged from 18 years to 30 years having the average experience of 2 years, and eight months were randomly selected from the registered active sports clubs and fitness gyms from three cities of Pakistan. The age groups of all participants included; 18-21 years $(\mathrm{n}=78,39.2 \%), 22-24$ years $(\mathrm{n}=66,33.2 \%), 25-27$ years $(\mathrm{n}$ $=30,15.1 \%), 28-30$ years $(n=25,12.6 \%)$. The participants' activities were as follows; soccer $(\mathrm{n}=22,11.1 \%)$, cricket $(\mathrm{n}=26,13.1 \%)$, filed hockey $(\mathrm{n}=4,2 \%)$, fitness gym $(\mathrm{n}=140,70.4 \%)$, badminton $(\mathrm{n}=1,0.5 \%)$, other sports $(\mathrm{n}=6,3 \%)$. Their experience (years) in sport/exercise participation was as follows; $1-2$ years $(\mathrm{n}=62,31.2 \%), 3-4$ years $(\mathrm{n}=84,42.2 \%), 7-8$ years $(\mathrm{n}=28,14 \%)$. The level of participation in sport competitions was as follows; city/club $(\mathrm{n}=167,84.9 \%)$, district $(\mathrm{n}=15,7.5 \%)$, provincial $(\mathrm{n}=13,6.5 \%)$, national $(\mathrm{n}=1,0.5 \%)$, others $(\mathrm{n}=1$, $0.5 \%)$.

Twenty four participants left some portions of the questionnaire incomplete or withdrawn from the participation and hence excluded from the study. The final sample consisted of 199 regular exercises and data obtained from these participants was included for analysis. Inclusion criteria for participants were as follows; a permanent member of a fitness gym or registered sports club minimum from one year, healthy adults having the ages from 18 years to 30 years, male gender, and exercise minimum one to two days per week at least during last three months. All participants voluntarily participated in this study.

\subsection{Procedure}

Lists of registered sports clubs and fitness gyms were obtained from the district sports office. One investigator approached personally to the coaches and managers of clubs and fitness gyms and requested for permission to collect data regarding sleep and exercise. Following this, the investigators discussed the feasible schedule for data collection 
with players and gym exercisers.

\subsection{Measures}

A questionnaire consisting of three sections including demographics, Pittsburg sleep quality index (PSQI), and sports and exercise-related questions was administered to obtain data from selected participants. All participants were instructed that their participation in this research was voluntary and the obtained information will be kept secret and confidential. Approximately 35 minutes were spent on filling up each questionnaire by each participant. Data collection was carried out during summer and hot weather conditions in May and June 2019.

\subsubsection{Demographics}

This section contains questions concerning participant's age, type of physical exercise, playing or exercise experience, level of exercise or sports participation, education, and socioeconomic status.

\subsubsection{Sleep Quality}

Participants' sleep quality was measured using the Pittsburg sleep quality index (PSQI) developed by Buysse, Reynolds III, Monk, Berman, and Kupfer (1989). This is a self-reported scale consisting of 21 items and seven components including "sleep quality, sleep latency, sleep duration, habitual sleep efficiency, sleep disturbances, use of sleeping medication, and daytime dysfunction"(Buysse et al., 1989). The global score is obtained by summing up the seven components of this scale. The individuals yielding a score of 5 or above considered poor sleepers. This scale is valid and reliable for measuring sleep quality in patients as well in healthy adults and older people and extensively used in sleep-related research (Mollayeva et al., 2016).

\subsubsection{Sports and Exercise}

Participants' sports and exercise frequency, intensity, and duration were measured using a validated and reliable three items questionnaire previously used by Cho, Kwon, and Jeon (2010); Park et al. (2016). For example, according to this questionnaire frequency was measured by asking the participants "During a week, how often do you participate in sports or physical exercise in your free time?". Responses were obtained on a five-point Likert scale as per procedure and exercise/ sports participation scores were computed in accordance with the procedure suggested by previous research (Park et al., 2016).

\section{Data Analysis}

Descriptive statistics were performed to determine the participant's characteristics and the prevalence of sleep problems among the selected sample. The data was not normally distributed thus the 2-tailed spearman correlation analysis considered appropriate to detriment relationship of physical exercises and sleep. SPSS software was used for data analysis.

\section{Results}

The spearman's rho (2-tailed) correlation test was performed for exercise, PSQI global score, and seven components of PSQI (subjective sleep quality, sleep latency, sleep duration, sleep efficiency, sleep disturbance, use of sleep medication, daytime dysfunction) to explore the relationship among these variables. A significant negative correlation was observed between the scores on the physical exercise measure and scores on sleep medication $(r=-.160, \mathrm{~N}=199$, $p<.05)$. This indicates that sleep medication score decreases as a result of increasing physical exercise suggesting a significantly positive relationship between physical exercise and sleep medication. The descriptive statistics yielded that $22 \%$ of the 199 participants exhibited the global score of PSQI of 5 or above suggesting the prevalence of sleep problems in approximately one-fourth of the total sample. On the other hand, it was also found that the average score of PSQI was 3.7 in total sample suggesting overall better sleep in the athletic population.

The result of the spearman's rho (2-tailed) between 199 exercisers yielded a significantly negative relationship between sports/ exercise participation level and sleep disturbance $(\mathrm{r}=-.149, \mathrm{~N}=199, p<.05)$. This suggests that increasing the level of sports/exercise participation results in decreasing sleep disturbance.

Exercise frequency was negatively correlated with daytime dysfunction $(\mathrm{r}=-.191, \mathrm{~N}=199, p<.01)$ whereas exercise intensity was negatively correlated with sleep disturbance $(\mathrm{r}=-.151, \mathrm{~N}=199, p<.05)$ and sleep efficiency $(\mathrm{r}=-.149$, 
$\mathrm{N}=199, p<.05)$ but positively correlated with daytime dysfunction $(\mathrm{r}=.150, \mathrm{~N}=199, p<.05)$. These suggest that increasing exercise frequency lead to decreases daytime dysfunction whereas increasing exercise intensity can significantly reduce sleep disturbance and improves sleep efficiency but increases daytime dysfunction.

The spearman's rho (2-tailed) test also yielded a significantly positive correlation between physical exercise and playing level $(r=.222, \mathrm{~N}=199, p<.005)$ suggesting that greater playing level leads to greater physical exercise. In addition, playing level and playing experience were significantly positively correlated with one another $(\mathrm{r}=.399, \mathrm{~N}=$ $199, p<.005)$.

Education level was significantly positively correlated with social status $(\mathrm{r}=.145, \mathrm{~N}=199, p<.05)$ and age $(\mathrm{r}=.296$, $\mathrm{N}=199, p<.001)$, and negatively correlated with physical exercise $(\mathrm{r}=-.170, \mathrm{~N}=199, p<.05)$. Moreover, there was a positive correlation of age positively with playing experience $(\mathrm{r}=.418, \mathrm{~N}=199, p<.001)$, and education level $(\mathrm{r}=$ $.296, \mathrm{~N}=199, p<.001)$. The analysis also found a positive correlation between ages and sleep efficiency $(\mathrm{r}=.159, \mathrm{~N}$ $=199, p<.05$ ) suggesting reduction in sleep efficiency with increased age among adult exercisers.

\section{Discussions}

This study investigated the relationship of physical exercise among male regular exercises in order to add valuable information for practical implications to deal with the problem of sleep among the masses. The analysis yielded various novel and meaningful findings. The findings showed a significantly negative relationship of physical exercise with sleep medication and a significantly negative relationship between sports/exercise participation level and sleep disturbance. These findings suggest a positive influence of physical exercise on sleep-related problems. It was also found that the average score of PSQI was 3.7 in total sample suggesting overall better sleep quality. On the other hand, one-fourth $(22 \%)$ of the total sample had a score of 5 or above suggesting the existence of sleep problems among regular exercisers. The findings further indicated that increased exercise frequency leads to decreases daytime dysfunction whereas increased exercise intensity can significantly reduce sleep disturbance and improves sleep efficiency but increasing daytime dysfunction. In general, these findings suggest the beneficial effect of exercise on sleep. Thus, physical exercise can be considered as a means to treat and prevent poor sleep among male adult exercisers. However, this problem still needs to address while looking for other factors that contribute to the prevalence of poor sleep among $22 \%$ male adult exercisers.

Concerning the findings of the relationship between physical exercise and sleep, it was found that physical exercise exerts a beneficial effect on sleep quality in terms of reducing sleep medication and sleep disturbance. This result supported by past research conducted by Brand et al. (2010b); Driller, Dixon, and Clark (2017); Rubio-Arias, MarínCascales, Ramos-Campo, Hernandez, and Pérez-López (2017) showing the effectiveness of physical exercise for better sleep for adolescents, student-athletes and middle-aged women. Although, some studies failed to show similar effects of physical exerciser for sleep (Driver \& Taylor, 2000), and the underlying reasons for the contradicting results are unknown. The discrepancies found regarding the relationship of physical exercise with sleep possibly linked with participant's demographic variables and characteristics that may underlie the different effects of physical exercise in the varying types of populations. Or it may cause by different methodologies and measuring tools (subjective versus objective measures) used in different studies.

Various explanations can be presented for the favorable effect of physical exercise for sleep in regular adult exercisers observed in this study. Exercise with moderate to high intensity appeared to enhance slow-wave sleep, minimizes sleep onset latency, and decreases awakening rate during night time sleep (Brand et al., 2010a), indicating that physical exercise possibly regulates homeostatic that further may lead to sleep regulation. Another possible reason might be that physical exercise improves negative psychological conditions and foster positive psychological functioning that indirectly led to improve sleep among exercises (Gerber et al., 2014). In addition, regular exercise suggested improving self-control, adaptive behaviors, and a healthy lifestyle (Ali, Azam, Mattiullah, \& Akhtar, 2019; Teixeira, Carraça, Markland, Silva, \& Ryan, 2012) that might collectively exert a positive impact on sleep among adult athletic population.

Moreover, the findings also revealed that increased exercise frequency leads to a decrease in daytime dysfunction whereas increased exercise intensity can significantly reduce sleep disturbance and improves sleep efficiency but 
increases daytime dysfunction. These likely to suggest that enhanced frequency and intensity of physical exercise exerts a positive influence on sleep by improving daytime dysfunction, sleep disturbance, and sleep efficiency. On the other hand, greater exercise intensity exerts a negative impact on daytime dysfunction. The effectiveness of enhanced exercise intensity for three PSQI components and negative effects on one component shows conflicting findings. Even though we are unsure about inferring the final conclusion, however, this data provides some support in favor of beneficial effects for enhanced exercise frequency and intensity for sleep. Further studies indeed required to clarify the contradicting results regarding relationships among exercise intensity and sleep components.

This finding seems congruent with the studies indicating more beneficial effects of moderate or vigorous exercise than that of exercise with low intensity. For example, Brand et al. (2014) found that moderate-intensity exercise improved sleep whereas low-intensity exercise showed no effectiveness for sleep quality. (Gerber et al., 2014) reported that exercise with high intensity was associated with better sleep in young adults. The possible reasons for this result might be that exercise with high intensity suggested increasing exertion that possibly favors deep sleep, reduces awakening along with decreasing sleep onset time (Brand et al., 2014) and these might result in healthy sleep. Another possible explanation might be that high-intensity exercises likely to protect against stress, depression, and other mental health issues (Gerber et al., 2014). These might further offset the negative influence of the factors that exert a negative impact on sleep and thus is likely to improve sleep health.

Another important finding that emerged from the analysis was that the average global score of PSQI was 3.7 for the total sample. According to the PSQI manual, the global score 4 or below indicates good and healthy sleep and PSQI global score of 5 or above indicates poor sleep (Buysse et al., 1989). Thus, this suggests that the quality of sleep in adult exercisers was good. This result seems in line with the main findings of this study discussed above showing a significantly positive relationship between exercise and sleep as well as positive association with exercise intensity and sleep. In contrast to this study, a study conducted by Swinbourne, Gill, Vaile, and Smart (2016) showed a 5.9 average score using PSQI measure in elite athletes suggesting a high prevalence of poor sleep in elite and professional athletes. The reason for the conflicting results of this study and previous research might be that there is a difference between levels of athletes. This study used athletes from local clubs and fitness gyms that likely to face fewer challenges and less competitiveness in comparison with elite athletes. It is likely that a tough schedule of sports competitions (Erlacher, Ehrlenspiel, Adegbesan, \& Galal El-Din, 2011) and excessive fatigue due to hard training schedules (Sargent, Lastella, Halson, \& Roach, 2014) can contribute sleep problems in elite athletes. But in the case of this study, athletes used as the sample in this study might face less challenging training schedules, less tough competitions that possibly contributed a favorable average score of PSQI measure in this study.

Even though the average PSQI score was below 4 in the total sample, however, the analysis yielded that approximately $22 \%$ of participants exhibited the score 5 or above suggesting the prevalence of poor sleep quality in one-fourth of the total sample of exercisers in this study. This result seems to contradict with above discussion regarding the beneficial effects of exerciser for sleep and better average PSQI score in the overall sample. One reason for this result might that the prevalence of sleep problems might be higher in the general population from where the sample of exercisers was selected. This view is supported by the research showing prevalence of sleep problems in $39.5 \%$ (Surani et al., 2015) or 31.3\% (Kidwai \& Ahmed, 2013) of the general population in the same country. These findings suggest that the rate of sleep problems comparatively lower in the adult exercisers used as a sample in this study compared with the prevalence of sleep problems in the general population in the same country. The lower prevalence level of sleep problems in the sample of this study suggests that physical exercise might play the role in comparatively lower rate of poor sleepers. Lastly, the prevalence of poor sleep observed in this study suggests that even though physical exercise found to be effective to improve sleep quality, there is a need to investigate the other factors that are underlying the reported level of poor sleep among adult exercises in this study. It is suggested to educate and spread awareness about sleep hygiene and the importance of healthy sleep patterns among adult exercisers.

\section{Limitations and future research}

In sum, this study has an important contribution by exploring the positive associations between physical exercise and sleep, beneficial effects of increased exercise frequency and intensity for sleep, good average sleep score in the total selected sample, and identifying the prevalence of poor sleep in the adult male exercisers from Pakistan. Based on 
these findings, there is a possibility to recommend physical exercise as a means to improve sleep and suggesting that exercise with high intensity and frequency should be preferred to improve sleep in male adults. On the other hand, the existence of sleep problems in the athletic population essentially requires further attention for reducing the prevalence of poor sleep using other approaches (i.e., sleep education) along with continuing exercise for good sleep.

Conclusively, these findings provide evidence to encourage adults for regular participation in sports activities and the development and promotion of sports facilities for sleep health.

\section{References}

Ali, A., Azam, M., Mattiullah, J., \& Akhtar, A. (2019). A systematic synthesis of evidence regarding relationship of physical activity and sports participation with trait self-control. Journal of Business and Social Review in Emerging Economies, 5(1), 19-40.

Anderson, E., \& Durstine, J. L. (2019). Physical Activity, Exercise, and Chronic Diseases: A Brief Review. Sports Medicine and Health Science. doi: https://doi.org/10.1016/j.smhs.2019.08.006

Bhaskar, S., Hemavathy, D., \& Prasad, S. (2016). Prevalence of chronic insomnia in adult patients and its correlation with medical comorbidities. Journal of family medicine and primary care, 5(4), 780-784. doi: 10.4103/22494863.201153

Brand, S., Gerber, M., Beck, J., Hatzinger, M., Pühse, U., \& Holsboer-Trachsler, E. (2010a). Exercising, sleep-EEG patterns, and psychological functioning are related among adolescents. The World Journal of Biological Psychiatry, 11(2), 129-140. doi: 10.3109/15622970903522501

Brand, S., Gerber, M., Beck, J., Hatzinger, M., Pühse, U., \& Holsboer-Trachsler, E. (2010b). High exercise levels are related to favorable sleep patterns and psychological functioning in adolescents: a comparison of athletes and controls. Journal of Adolescent Health, 46(2), 133-141.

Brand, S., Kalak, N., Gerber, M., Kirov, R., Pühse, U., \& Holsboer-Trachsler, E. (2014). High self-perceived exercise exertion before bedtime is associated with greater objectively assessed sleep efficiency. Sleep Medicine, 15(9), 1031-1036. doi: https://doi.org/10.1016/j.sleep.2014.05.016

Buysse, D. J., Reynolds III, C. F., Monk, T. H., Berman, S. R., \& Kupfer, D. J. (1989). The Pittsburgh Sleep Quality Index: a new instrument for psychiatric practice and research. Psychiatry research, 28(2), 193-213.

Cao, X.-L., Wang, S.-B., Zhong, B.-L., Zhang, L., Ungvari, G. S., Ng, C. H., . . Xiang, Y.-T. (2017). The prevalence of insomnia in the general population in China: A meta-analysis. PLoS ONE, 12(2), e0170772. doi:

10.1371/journal.pone.0170772

Caspersen, C. J., Powell, K. E., \& Christenson, G. M. (1985). Physical activity, exercise, and physical fitness: definitions and distinctions for health-related research. Public health reports, 100(2), 126.

Cho, M., Kwon, W.-D., \& Jeon, Y.-B. (2010). Are Korean secondary school girls physically active during leisure time? Health care for women international, 31(3), 244-257.

Driller, M. W., Dixon, Z. T., \& Clark, M. I. (2017). Accelerometer-based sleep behavior and activity levels in student athletes in comparison to student non-athletes. [journal article]. Sport Sciences for Health, 13(2), 411-418. doi: 10.1007/s11332-017-0373-6

Driver, H. S., \& Taylor, S. R. (2000). Exercise and sleep. Sleep medicine reviews, 4(4), 387-402.

Erlacher, D., Ehrlenspiel, F., Adegbesan, O. A., \& Galal El-Din, H. (2011). Sleep habits in German athletes before important competitions or games. Journal of sports sciences, 29(8), 859-866. doi:

10.1080/02640414.2011.565782

Gerber, M., Brand, S., Herrmann, C., Colledge, F., Holsboer-Trachsler, E., \& Pühse, U. (2014). Increased objectively assessed vigorous-intensity exercise is associated with reduced stress, increased mental health and good objective and subjective sleep in young adults. Physiology \& Behavior, 135, 17-24.

Kidwai, R., \& Ahmed, S. H. (2013). Prevalence of insomnia and use of sleep medicines in urban communities of Karachi, Pakistan. Journal of Pakistan Medical Association, 63(11), 1358-1363.

Mollayeva, T., Thurairajah, P., Burton, K., Mollayeva, S., Shapiro, C. M., \& Colantonio, A. (2016). The Pittsburgh sleep quality index as a screening tool for sleep dysfunction in clinical and non-clinical samples: a systematic review and meta-analysis. Sleep medicine reviews, 25, 52-73.

Mork, P. J., Vik, K. L., Moe, B., Lier, R., Bardal, E. M., \& Nilsen, T. I. L. (2013). Sleep problems, exercise and obesity and risk of chronic musculoskeletal pain: the Norwegian HUNT study. The European Journal of Public Health, 24(6), 924-929.

Park, J.-A., Park, M.-H., Shin, J.-H., Li, B., Rolfe, D. T., Yoo, J.-Y., \& Dittmore, S. W. (2016). Effect of sports 

participation on internet addiction mediated by self-control: A case of korean adolescents. Kasetsart Journal of Social Sciences, 37(3), 164-169.

Rubio-Arias, J. Á., Marín-Cascales, E., Ramos-Campo, D. J., Hernandez, A. V., \& Pérez-López, F. R. (2017). Effect of exercise on sleep quality and insomnia in middle-aged women: A systematic review and meta-analysis of randomized controlled trials. Maturitas, 100, 49-56. doi: https://doi.org/10.1016/j.maturitas.2017.04.003

Sargent, C., Lastella, M., Halson, S. L., \& Roach, G. D. (2014). The impact of training schedules on the sleep and fatigue of elite athletes. Chronobiology International, 31(10), 1160-1168. doi: 10.3109/07420528.2014.957306

Singareddy, R., Vgontzas, A. N., Fernandez-Mendoza, J., Liao, D., Calhoun, S., Shaffer, M. L., \& Bixler, E. O. (2012). Risk factors for incident chronic insomnia: A general population prospective study. Sleep Medicine, 13(4), 346-353. doi: https://doi.org/10.1016/j.sleep.2011.10.033

Surani, A. A., Zahid, S., Surani, A., Ali, S., Mubeen, M., \& Khan, R. H. (2015). Sleep quality among medical students of Karachi, Pakistan. J Pak Med Assoc, 65(4), 380-382.

Swinbourne, R., Gill, N., Vaile, J., \& Smart, D. (2016). Prevalence of poor sleep quality, sleepiness and obstructive sleep apnoea risk factors in athletes. European Journal of sport science, 16(7), 850-858. doi: 10.1080/17461391.2015.1120781

Teixeira, P. J., Carraça, E. V., Markland, D., Silva, M. N., \& Ryan, R. M. (2012). Exercise, physical activity, and selfdetermination theory: A systematic review. International journal of behavioral nutrition and physical activity, 9(1), 78. doi: 10.1186/1479-5868-9-78

Zschucke, E., Gaudlitz, K., \& Ströhle, A. (2013). Exercise and physical activity in mental disorders: clinical and experimental evidence. Journal of preventive medicine and public health = Yebang Uihakhoe chi, 46 Suppl 1(Suppl 1), S12-S21. doi: 10.3961/jpmph.2013.46.S.S12 